An Effective Model for

Dynamic Finite Difference Calculations

T. N. Dey 



\section{DISCLAIMER}

This report was prepared as an account of work sponsored by an agency of the United States Government. Neither the United States Government nor any agency thereof, nor any of their employees, makes any warranty, express or implied, or assumes any legal liability or responsibility for the accuracy, completeness, or usefulness of any information, apparatus, product, or process disclosed, or represents that its use would not infringe privately owned rights. Reference herein to any specific commercial product, process, or service by trade name, trademark, manufacturer, or otherwise does not necessarily constitute or imply its endorsement, recommendation, or favoring by the United States Government or any agency thereof. The views and opinions of authors expressed herein do not necessarily state or reflect those of the United States Government or any agency thereof. 


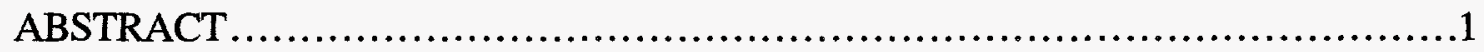

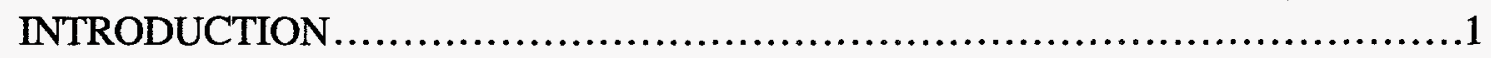

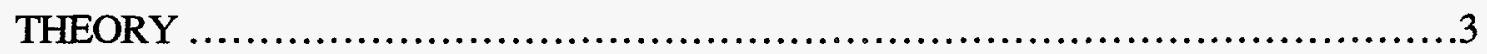

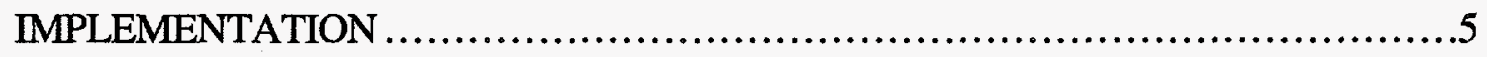

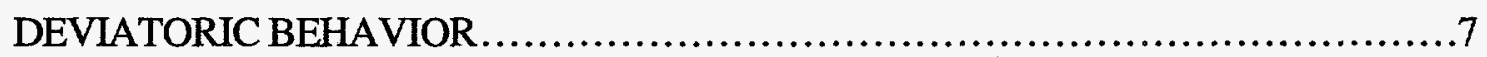

ARTIFICIAL VISCOSITY .................................................10

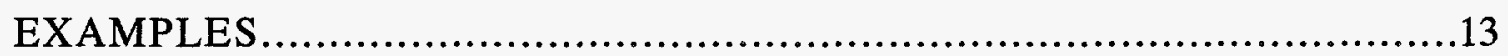

Example 1 - Simulation of a quasistatic laboratory experiment....................13

Example 2 - $1 \mathrm{kt}$ explosion in fully and partially saturated limestone...............15

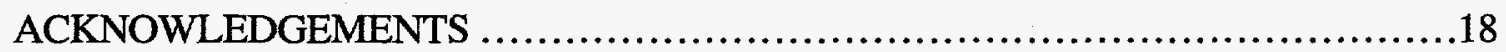

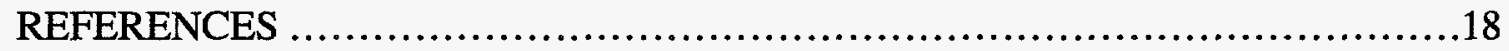

Appendix A - SMC123 Implementation...........................................21

Appendix B - CTH Implementation .......................................... 

pore fluid through the solid matrix. The effective stress rule they chose to impose was that the volume fraction occupied by the solid is strictly a function of the difference between the total mean stress and the pore fluid pressure, which is the conventional definition of effective pressure. Note that this assumption is equivalent to assuming that the total porosity is a function of only the effective pressure. In this work, quadratic polynomials were used to fit the volume fraction-effective pressure data taken in experiments where the pore pressure was held at zero, but no attempt was made to account for the hysteresis in this pore crushing relationship. Garg and co-workers examined the influence of the relative motion of the pore fluid and the solid matrix as a function of permeability and fluid viscosity and noted the strong dissipation that can occur under some conditions.

Swift and Burton (1984) developed an effective stress model for use on dynamic and quasistatic problems, and implemented this model in the TENSOR finite-difference code. Their model was based on mixture theory and generally follows the development of Garg described above. It is assumed that the total porosity is function of the effective pressure. The numerical implementation is specified in an incremental form and uses equations-of-state (EOSs) for the solid and fluid components. The effects of pore crushing are introduced by casting the porosity into a form where it is a function of the drained bulk modulus. Hysteresis in the hydrostatic pore compaction as well as additional effects such as shear enhanced compaction are introduced through the behavior of the drained bulk modulus. This method of handling shear enhanced pore compaction is to be contrasted to the more general and more complicated method of introducing this effect through the flow rule for shear failure.

Carroll and co-workers (Carroll, 1980; Carroll and Holt, 1972; Katsube and Carroll, 1987a, b; and Katsube, 1985) developed a phenomenological model which was motivated by the work of Biot (1941) and of Nur and Byerlee (1971). They show that the deformation of a fluid-filled poroelastic solid can be decomposed into three components: the deformation of the solid, the volume change of the fluid, and the change in pore geometry. They point out that there is no guarantee that, in a conventional mixture theory, the effective deformations will be related to the effective stresses through the constitutive law of the components. They introduce the micromechanical assumption that the volume average stress equals the sums of the porosity weighted volume average stresses of the components, $\sigma_{i j}=\phi \sigma_{i j}^{\text {fluid }}+(1-\phi) \sigma_{i j}^{\text {solid }}$, where $\sigma_{i j}$ is the respective stress tensor and $\phi$ is the porosity defined as the ratio of the pore volume to the total volume of the representative element of material. Making this assumption in a mixture theory overcomes their concern about the constitutive laws of the components and results in a theory which is equivalent to their phenomenological model. 


\title{
AN EFFECTIVE STRESS MODEL FOR DYNAMIC FINITE DIFFERENCE CALCULATIONS
}

by

T.N. Dey

\begin{abstract}
An effective stress model, which simulates the mechanical effects of pore fluids on deformation and strength of porous materials, is described. The model can directly use SESAME table equations-ofstate (EOSs) for the solid and fluid components. The model assumes that undrained (no fluid flow) conditions occur. Elastic and crushing behavior of the pore space can be specified from the results of simple laboratory tests. The model fully couples deviatoric and volumetric behavior in the sense that deviatoric and tensile failure depend on the effective pressure, while volumetric changes caused by deviatoric failure are coupled back to the volumetric behavior of the material. Strain hardening and softening of the yield surface, together with a number of flow rules, can be modeled. This model has been implemented into the SMC123 and CTH codes.
\end{abstract}

\section{INTRODUCTION}

The deformation of geological materials such as rock and soil is complicated by the fact that these materials generally have some porosity and this porosity is often partially or fully saturated by water. Fortunately, the behavior of a wide variety of these materials follows effective stress relationships like those defined by Terzaghi (1943). Such relationships can form the basis of a numerical model for volumetric and shear deformation of materials under static and dynamic loading conditions. A number of such numerical models have been developed and implemented in codes suitable for calculating stress wave propagation and deformation due to explosions or impacts.

The work of Biot (1941) is a well known analytic model for consolidation and pore fluid flow under quasi-static infinitesimal strain conditions. Garg and co-workers (Garg, 1971; Garg, Nayfeh and Good, 1974; Garg et al., 1975) developed a model for the volumetric behavior suitable for numerical computations and finite strain conditions. Their work was based on a mixture theory approach and allowed for the simultaneous flow of the 
Other models, suitable for use in ground shock calculations, are those of Sandler and Whitman (1990), Frederickson, et al (1989), and Kim, et al (1988). These models are cast in an incremental form, are valid for undrained conditions, and are generally similar to the Burton and Swift model described above. Simons (1989) has compared these three models with those of Burton and Swift, Carroll, and the model described in this report. $\mathrm{He}$ finds differences that will only cause negligible differences in results for the typical use of these models. Practical considerations, which users must judge for themselves, may or may not give preference to some of these models over others.

The model described in this report is based on this phenomenological model of Carroll and co-workers. The natural separation of the deformation into three components provided by this approach allows the use of conventional EOSs for the solid and fluid components as well as allowing a straightforward reduction of laboratory data on pore compaction into a form suitable for use with the model.

\section{THEORY}

The first of two key assumptions in the model is the micromechanical assumption that the pores in the material are distributed at random spatially such that the areal porosity equals the volumetric porosity. This assumption does not require that the pores have random orientations. Following the development of Carroll and others described earlier, this allows volumetric averages of the stresses to be written as

$$
\sigma_{i j}=\phi \sigma_{i j}^{\text {fluid }}+(1-\phi) \sigma_{i j}^{\text {solid }}
$$

This equation follows from the assumption that the fraction of any cross section acted on by the fluid stress is $\phi$ and that the solid stress acts on the remaining fraction. The fluid is assumed to only support pressure, giving $\sigma_{i j}^{\text {fluid }}=-P_{f l u i d} \delta_{i j}$ and the mean stress component of (1) can be written as:

$$
P_{\text {total }}=\phi P_{\text {fluid }}+(1-\phi) P_{\text {solid }}
$$

The fluid and solid are assumed to follow their respective EOSs:

$$
\begin{aligned}
& P_{\text {fluid }}=P_{\text {fluid }}\left(\rho_{\text {fluid }}, E_{\text {fluid }}\right)=P_{\text {fluid }}\left(m_{\text {fluid }} /(\phi V), E_{\text {fluid }}\right) \\
& P_{\text {solid }}=P_{\text {solid }}\left(\rho_{\text {solid }}, E_{\text {solid }}\right)=P_{\text {solid }}\left(m_{\text {solid }} /((1-\phi) V), E_{\text {solid }}\right)
\end{aligned}
$$


The densities of the fluid and solid are defined consistently with the phenomenological formulation, namely, the fluid density is the mass of fluid in a representative volume of material divided by the pore volume in that representative volume while the solids density is the mass of solid divided by the difference between the total representative volume and its corresponding pore volume. The specific internal energy of the fluid is determined by accumulating the work done on the fluid over the course of the calculation. The specific internal energy of the solid is determined from the difference between the internal energy of the complete material and the internal energy of the fluid. Note that for a partially saturated material at room conditions, the fluid density definition in equation (3) will give a density for the pore fluid that is less than its usual room condition value. The fluid EOS is required to give only the vapor pressure of the fluid under this condition. This requirement allows both saturated and unsaturated conditions to be treated in the model, and no distinction needs to be made between these conditions. This density definition leads to the relationship that

$$
\rho=\phi \rho_{\text {fluid }}+(1-\phi) \rho_{\text {solid }}
$$

The second key assumption is that the porosity depends only on the effective pressure:

$$
\phi=\phi\left(P_{\text {eff }}\right)=\phi\left(P_{\text {total }}-P_{\text {eff }}\right)
$$

and possibly some history-dependent internal variables, including porosity corrections due to shear enhanced void collapse or shear induced dilatancy. At the present time, no attempt is being made to force temperature equilibrium between the fluid and the solid. This last assumption completes the set of equations required to solve for all the necessary quantities given the density of the material and the specific energies of the solid and fluid components.

Carroll and co-workers specifically define the EOS response of the fluid and solid to be a linear elastic material. This enables them to proceed on to analytic solutions under a variety of conditions and compare these solutions to previous work, notably that of Biot. For the applications for which the current model is intended, shock wave propagation and penetration mechanics in soils and rocks, nonlinear behavior will be important. Consequently, the model has been defined such that any reasonable EOSs can be implemented for the fluid and the solid. The set of equations above is to be solved numerically, as detailed in the next section. 


\section{IMPLEMENTATION}

Equations (2), (3), (4), and (6) above provide the required information for carrying out a numerical simulation of the volumetric behavior. In a typical explicit time-stepping finite difference code, the density and internal energy of a material are known, and the corresponding stress state is to be determined. The procedure adopted here is to rewrite (2) with the help of the definition of the effective pressure as

$$
P_{\text {eff }}=(\phi-1) P_{\text {fluid }}+(1-\phi) P_{\text {solid }}
$$

Equation (6) is inverted to

$$
P_{\text {eff }}=\mathrm{f}(\phi)
$$

Taking the difference between these two equations leaves an equation with the porosity $\phi$ as the independent variable:

$$
\mathrm{f}(\phi)=(\phi-1) P_{\text {fluid }}\left(m_{\text {fluid }} /(\phi V), E_{\text {fuid }}\right)+(1-\phi) P_{\text {solid }}\left(m_{\text {solid }} /((1-\phi) V), E_{\text {solid }}\right)
$$

This equation is solved iteratively using Newton's method together with a convergence accelerator described by Boettger and Trickey (1984). The discontinuity in slope of the fluid EOS as porosity changes between values giving saturated behavior and unsaturated behavior can cause the straightforward application of Newton's method to converge very slowly when the solution is near this point. The additional use of the convergence accelerator overcomes this and allows convergence in only a few iterations, even under these circumstances.

The effective pressure-porosity relationship is described by a table. A plot of such a table for an $11 \%$ porosity Indiana limestone is shown in Figure 1. The table contains an initial crush curve and an unloading curve from the highest point on the initial crush curve. Unloading from peak effective pressure values other than that for the master unloading curve is constructed by shifting a line tangent to the initial crush curve and the entire master unloading curve in porosity until both intersect the desired peak effective pressure on the initial crush curve. A weighted sum of these two shifted curves is used as the unloading curve from this point. The weighting is formed by taking the difference of the normalized 
porosity at point $\mathrm{A}$ on the plot below and the minimum normalized porosity experienced by the cell. This difference is divided by the difference in normalized porosities at points B and $A$ in the figure.

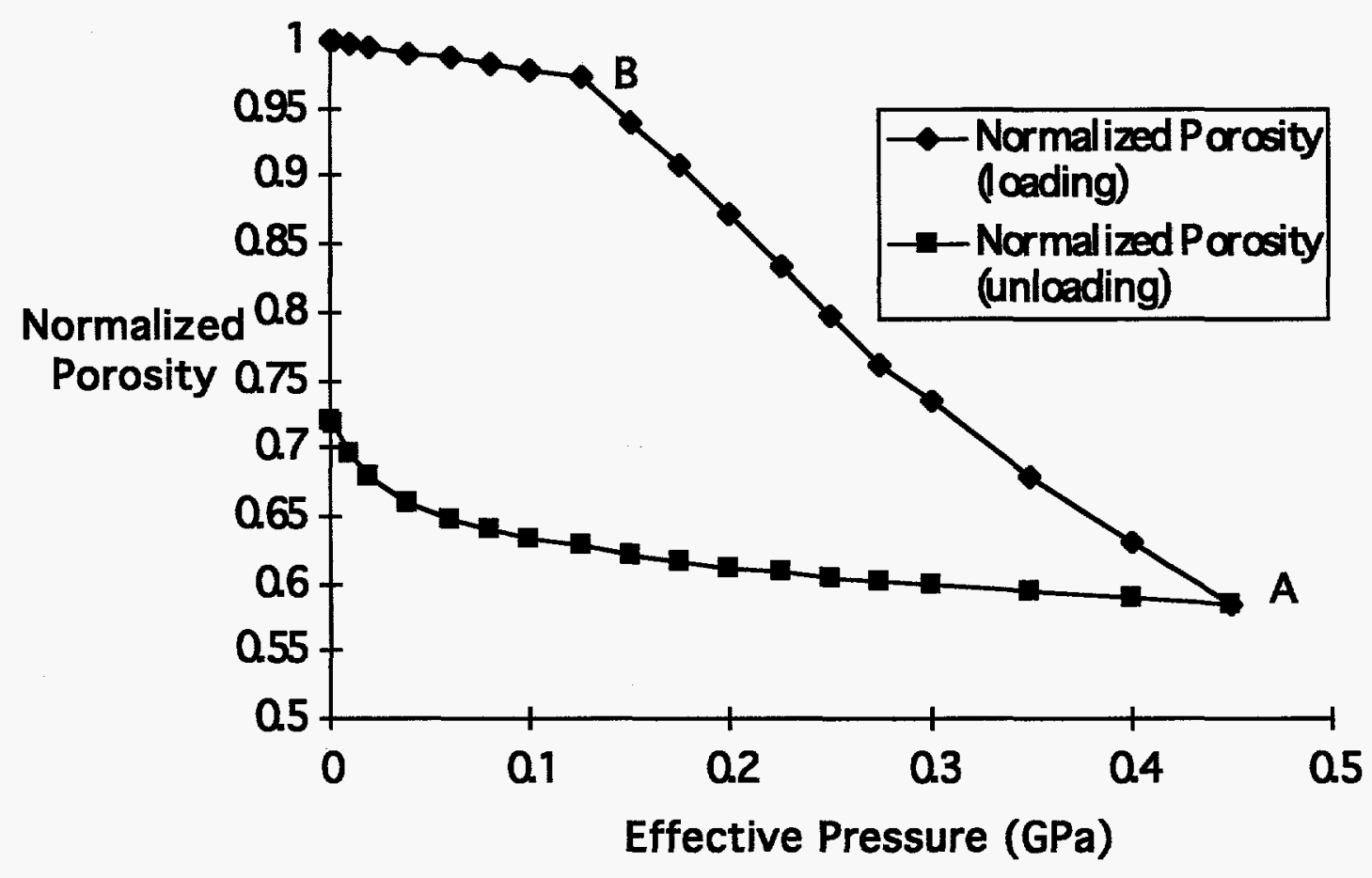

Figure. 1. Example of effective pressure-normalized porosity data.

This ratio is clipped to the range 0 to 1 and then taken to the power corresponding to a user specified exponent. This computation is specified by the equation

$$
\mathrm{w}=\left(\frac{\bar{\phi}-\bar{\phi}_{A}}{\bar{\phi}_{B}-\bar{\phi}_{A}}\right)^{\mathrm{n}} \text { for } \bar{\phi}_{A} \leq \bar{\phi} \leq \bar{\phi}_{B}
$$

where $\bar{\phi}$ is the minimum normalized porosity. For virgin material, this weighting factor is initially 1 . As the minimum normalized porosity approaches point $\mathrm{A}$, the weighting factor goes to zero. With an exponent of 1 , the weighting factor varies linearly between points $B$ and $\mathrm{A}$. With an exponent much greater than 1 , the weighting factor goes to zero quickly as the normalized porosity proceeds from point $\mathrm{B}$ to point $\mathrm{A}$ resulting in the interpolated unload curve having a shape very close to that of the master unload curve. For an exponent much smaller than 1 , the weighting factor remains close to 1 until the minimum normalized 
porosity is nearly at point A so most interpolated unload curves will be straight lines parallel to the initial compression slope. This construction requires saving the minimum normalized porosity experienced by a cell as an internal variable for the model. Porosities in the table are normalized by the initial porosity of the material making it simple to change initial porosities in a problem, and, as will be discussed later, simplifying the handling of porosity created or destroyed by yielding in shear.

The EOSs of the fluid and the solid components are described in the current implementation by either a SESAME table, a tabular EOS which covers a broad range of pressures and temperatures and typically includes the vaporization transition, or a simple pressure-volume table, which ignores the effects of internal energy and is suitable for problems with only small maximum compressions. Consequently, the model is capable of spanning a range of behavior from linear elastic up through the strong shock region were pressures may be many tens or hundreds of GPa. SESAME tables can accommodate equilibrium phase transformations, such as that at about $1 \mathrm{GPa}$ in calcite, so that the model can continue to work under these conditions.

Note, also, that the EOS of the fluid could be based on a mixture of a number of fluid components using a conventional mixture theory. This allows a relatively simple extension of this model to certain types of multicomponent problems such as mixtures of water, petroleum and natural gas. Additionally, since the model is formulated directly in terms of the mass of fluid in the representative volume, an extension of the model to conditions where relative flow of the pore fluid through the porous material occurs is straightforward under conditions where relative transport of momentum by the fluid is negligible.

\section{DEVIATORIC BEHAVIOR}

The method for treating deviatoric behavior is similar to that used in noneffective stress models. The two differences are that the yield surface is considered to be a function of the effective pressure and that porosity changes from dilatancy or pore collapse caused by yielding in shear are linked back into the porosity of the material. The onset of failure in rocks and soils is commonly observed to obey effective stress rule (Handin, et al., 1963, Nur and Byerlee, 1971; Brace, 1972; Jaeger and Cook, pp. 223-225, 1976). Typically, these materials also show a significant dependence of the compressive yield stress on pressure. The only modification needed, in this regard, for an effective stress model is to make the yield surface a function of the effective pressure instead of the total pressure, and this is the method used by the model.

An initial and an ultimate yield surface may be specified for the material, and the model interpolates between these two according to the equivalent plastic strain which has 
accumulated. Figure 2 shows an example of initial and ultimate yield surfaces for an $11 \%$ porosity Indiana limestone. The two yield surfaces may be arbitrarily stronger or weaker than each other, may cross each other, and do not need to vary monotonically with effective pressure. In the deviatoric plane (i.e., the $\pi$-plane or octahedral plane, which, in principal stress space, is normal to the line defined by $S_{1}=S_{2}=S_{3}$ ), the yield surface may have a shape varying from the conventional circle to a triangle. This option allows the model to represent the differences in strength observed under conditions of triaxial compression, triaxial extension and other general stress states.

The flow rule for the model also follows conventional practices. Since the deviatoric part of the model is an incremental model, the usual corrections, as appropriate for the desired flow rule, to the deviatoric part of the stress tensor are all that are required. Since the volumetric part of the model is not an incremental model, an additional step is required for any volumetric increment required by the flow rule. Phenomenologically, the volumetric increment represents void space created or destroyed by yielding in shear. A decision must be made as to how further hydrostatic components of loading will deform this void space. The choice made here is to use the volumetric increment to compute a correction to the initial porosity of the material. Since the porosity crush curve is specified using a normalized porosity, the increment to the initial porosity is automatically given the same crushing characteristics as the existing porosity. There is no particular theoretical justification for this assumption other than expediency.

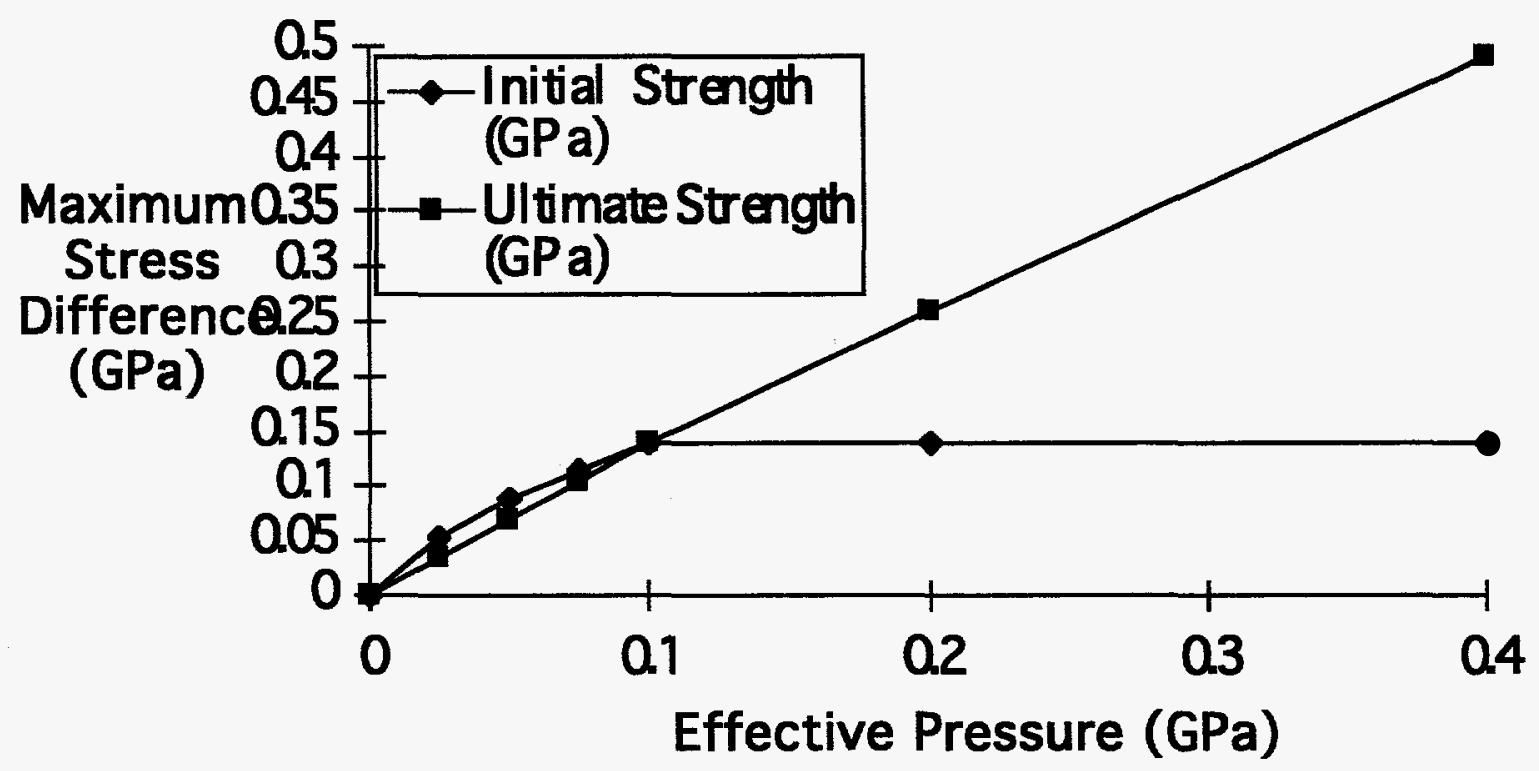

Figure 2. Initial and ultimate shear yield surfaces for an $11 \%$ porosity Indiana limestone. 
Three choices of flow rule are implemented with this model (Figure 3). The simplest is designated the Prandtl flow rule and is the most commonly used in many finite difference codes. Following the conventional practice of the radial return method of Wilkins (1964), a trial increment of the deviatoric stresses is made from the deviatoric strain increments assuming the material is behaving as a linear elastic material with a shear modulus determined from the present conditions of the material. If the deviatoric stress state exceeds the material yield surface, all the deviatoric stresses are multiplied by the same constant factor so that the new deviatoric stress state lies on the yield surface. No adjustments to the mean stress are made, so this flow rule gives no porosity change during yielding.

The second flow rule choice is the associated flow rule. After the elastic trial state is determined, both the mean effective stress and the deviator stresses are modified to bring the new deviatoric stress state to the yield surface. The ratio of the increment of the mean effective stress to the increment of the second invariant $\left(\mathrm{J}_{2}{ }^{\prime}\right)$ of the deviatoric stress tensor is equal to the slope of the yield surface in the $\mathrm{J}_{2}{ }^{\prime}-\mathrm{P}_{\text {eff }}$ plane. The increment in porosity consistent with the required mean effective stress increment is also computed, and the total porosity of the material is adjusted as described earlier.

The third flow rule choice is a simple, arbitrary choice of the ratio of the increment of the effective pressure to the increment of the second invariant of the deviatoric stress tensor. If this ratio is chosen to be zero, the first flow rule choice, described earlier, is obtained. As implemented in the model, this ratio is also allowed to change linearly with the factor used to interpolate between the initial and ultimate yield surfaces. 


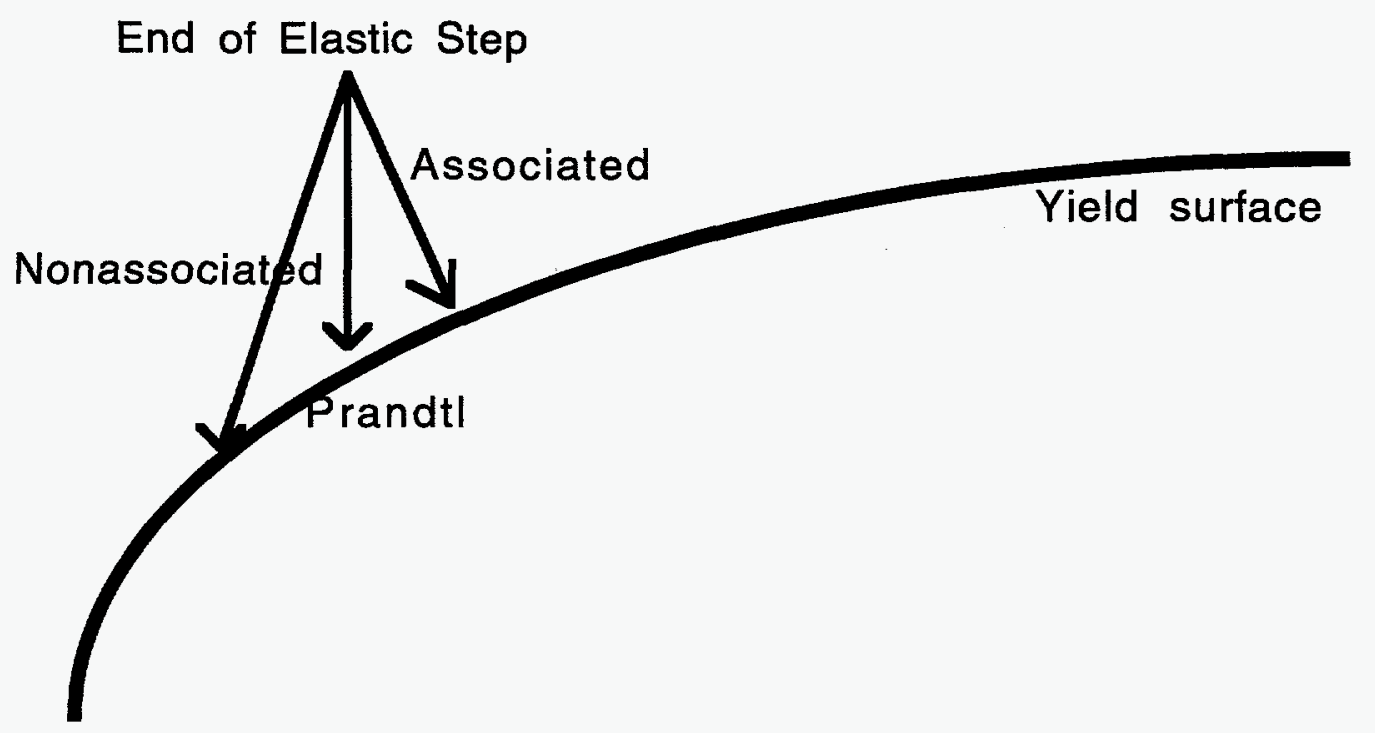

Figure 3. Yield stress as a function of effective pressure is plotted. The three options for returning to the yield surface from an elastic step outside the yield surface are shown.

Failure in tension is modified, compared to conventional total stress models, in a way similar to the way failure in shear is modified. Instead of comparing the principal values of the total stress tensor to the tensile strength, the principal values of the effective stress tensor are compared. According to this criterion, it is possible for a tensile failure to occur under conditions where all the total stress components are compressive. These conditions are those where a hydraulic fracturing type failure occurs due to the high pore fluid pressure.

Instead of directly computing the principal components of the effective stress tensor, the tensile analysis can be simplified by casting the tensile strength criterion into a yield surface with the critical value of the second invariant of the effective deviatoric stress tensor being expressed as a function of the effective pressure and the third invariant.

The code then compares this value with the shear yield surface value under the same conditions and uses the lesser value in the radial return algorithm. It is also necessary to test the effective pressure itself against the tensile strength value, and this test is built into the solver for the volumetric portion of the effective stress model.

\section{ARTIFICIAL VISCOSITY}

The conventional von Neumann-Richtmeyer artificial viscosity scheme generally works well when modeling shock wave propagation in materials with smoothly varying 
pressure-volume relationships. While one can think of many different ways in which to describe what artificial viscosity does, for the purposes of this model it is convenient to think about how it modifies the path in pressure-volume space as a shock passes a material point. In this space, the purpose of the artificial viscosity is to add sufficient additional pressure so that the path will roughly approximate a Rayleigh line. The conventional artificial viscosity function provides a good approximation for material models with smoothly varying $\mathrm{P}-\mathrm{V}$ curves. In porous materials, the $\mathrm{P}-\mathrm{V}$ behavior may be reasonably smooth while there is open void space remaining. The behavior usually stiffens abruptly as the last of the void space is closed. The conventional artificial viscosity scheme, with parameters chosen appropriately for typical non-porous materials, does not add sufficient viscous pressure for this case. Figure 4 shows an example. Substantially ringing in the wave form while unloading from the peak stress point, which appears as loops in the P-V curve, is the result.

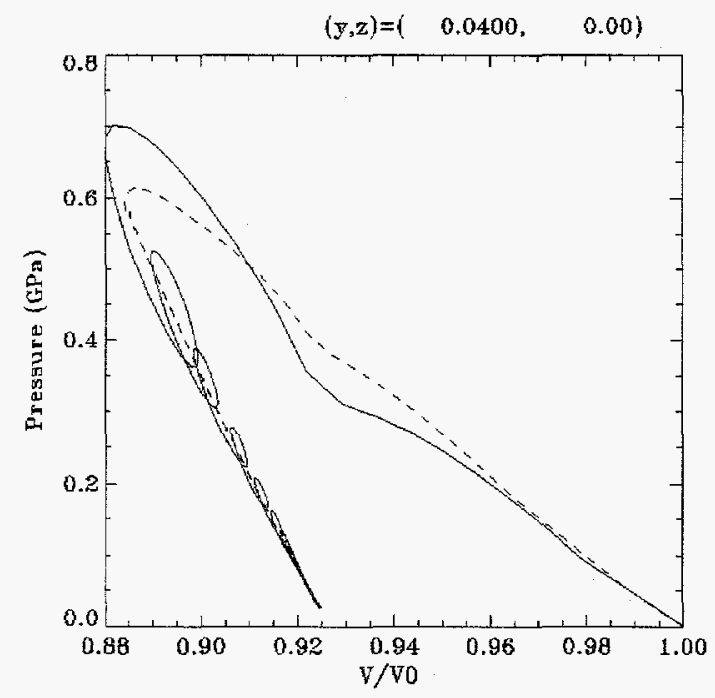

Figure 4. Path in pressure volume space for a material point during the passage of a shock. The result with the conventional artificial viscosity follows the solid line while the dashed line shows the result with the modified viscosity.

To mitigate this problem, the model contains a purely empirical correction to the conventional artificial viscosity. The correction is to multiply the linear artificial viscosity term by $(1+$ factor $)$ 
where factor $=$ qextra $\times \max \left(0, \min \left(1,1-a b s\left(\frac{\rho_{\text {norm }}-\rho}{\rho_{\text {norm }}-\rho_{0}}\right)\right)\right.$

$\rho$ is the current material density,

$\rho_{0}$ is the initial density, and

$\rho_{\text {norm }}$ is the normal density (ie. the density in the absence of any void space).

A value of 2 for the qextra parameter appears to work well. This functional form maximizes the viscosity increase just at the point where the last of the void space is crushed. The viscosity increase tapers linearly to zero for densities higher and lower than this. Figure 4 shows the improved P-V behavior as a result of applying this correction. A better approximation to the Rayleigh line is provided, and there are no oscillations visible as the material unloads.

Since the linear viscosity is proportional to the material sound speed, the simplest implementation, particularly in CTH, is simply to multiply the sound speed by the $(1+$ factor $)$ term. This generally has little effect on the time step in explosion problems since the high sound speed in the explosive typically controls the time step. This method has the advantage of allowing the artificial viscosity for nonporous materials to follow the conventional scheme.

Herrmann (1968) proposed an artificial viscosity change for materials with crushable void space. His scheme adds in additional viscous pressure only while void space is present. He uses a conventional viscous pressure form with a special set of linear and quadratic coefficients to do this. This scheme usually does provide an improvement over the conventional viscosity treatment. It does not create as consistently good an approximation to the Rayleigh line as the treatment described here, primarily because some additional viscous pressure is required during the shock passage during continued compression beyond that required to close the void space.

A further issue with artificial viscosity is how to properly distribute the additional work done by the stresses due to the artificial viscosity to each of the components in the material. The choice made here is to augment the solid pressure and the fluid pressure by the viscous pressure and compute the work done in changing the volume of that component. Actually, it is only necessary to compute the work done on the fluid, since the change in solid energy can be computed from the change for the whole material less the change for the fluid. While this is an ad hoc method of partitioning the work due to the artificial viscosity, it does satisfy obvious limiting cases. For fully saturated cases with $0 \%$ and $100 \%$ total porosity, the water will be assigned none and all of the internal energy respectively. For an incompressible solid, the water will also be assigned all the internal 
energy. When the water is more compressible than the solid, which is normally the case, the water will be assigned a higher specific internal energy, which is at least qualitatively correct.

\section{EXAMPLES}

\section{Example 1 - Simulation of a quasistatic laboratory experiment.}

Quasistatic laboratory experiments were performed by Blouin (Kim, et al., 1978) on samples of Salem, Indiana, limestone. Drained experiments were performed to give data for setting up effective stress models, and then undrained experiments were performed to give data for testing the models. The limestone samples contained $11 \%$ porosity. The effective stress model was set up using a user-supplied table for the limestone solids P-V relationship and SESAME Table 7157 for the pore water EOS. The effective pressureporosity table was constructed by digitizing the drained experiment hydrostat and then using the solids bulk modulus together with Equation (9) with the pore fluid pressure set to zero to solve for the porosity. A graph of the porosity table is displayed in Figure 1. The resulting fit together with the drained hydrostat data are shown in Figure 5. Resetting the pore water saturation in the model to $100 \%$ and rerunning the simulation then gives the model prediction of the undrained experiment as shown in Figure 6. The shear strength of this rock shows brittle behavior (weakening) for effective pressures below $100 \mathrm{MPa}$ and shows ductile behavior and strengthening for effective pressures greater than this. Figure 2 shows plots of the initial and ultimate shear yield surface used for this limestone. The material also displays shear-enhanced void collapse during yielding in shear, and this behavior is also modeled through the flow rule parameters. A non-associated flow rule option is required to simulate this behavior.

This example was run using the SMC123 code. The namelists in the input file which describe the material model are shown below. Appendix A gives a description of each of the parameters.

The following namelist provides the description of the volumetric portion of the effective stress model for the limestone in this example:

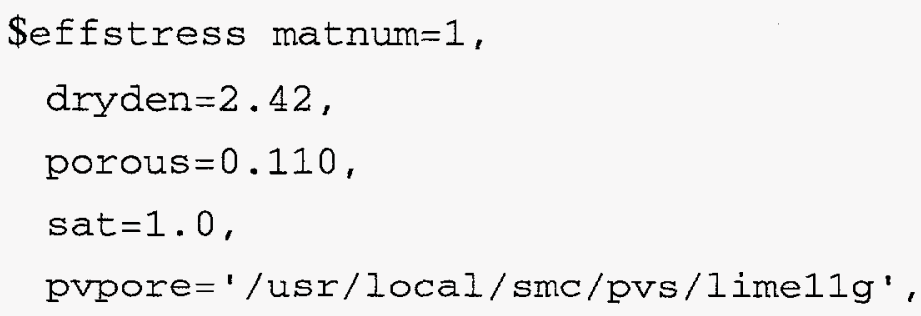




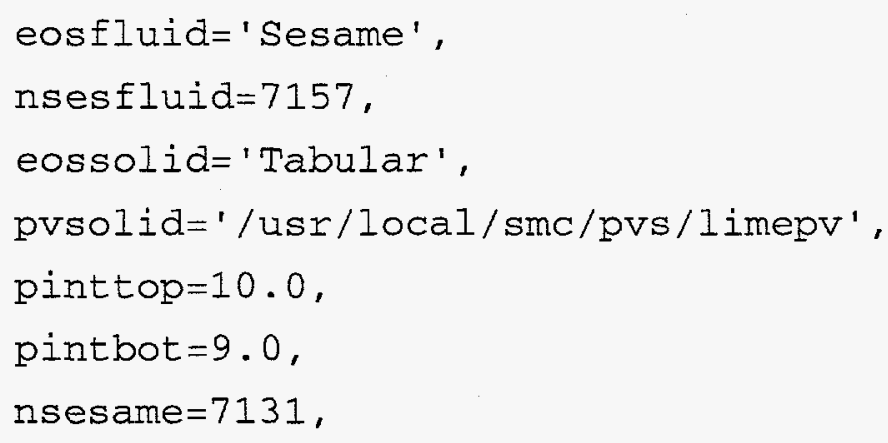

The following namelist provides the description of the tensile and shear strengths, strength damage, and flow rule behavior:

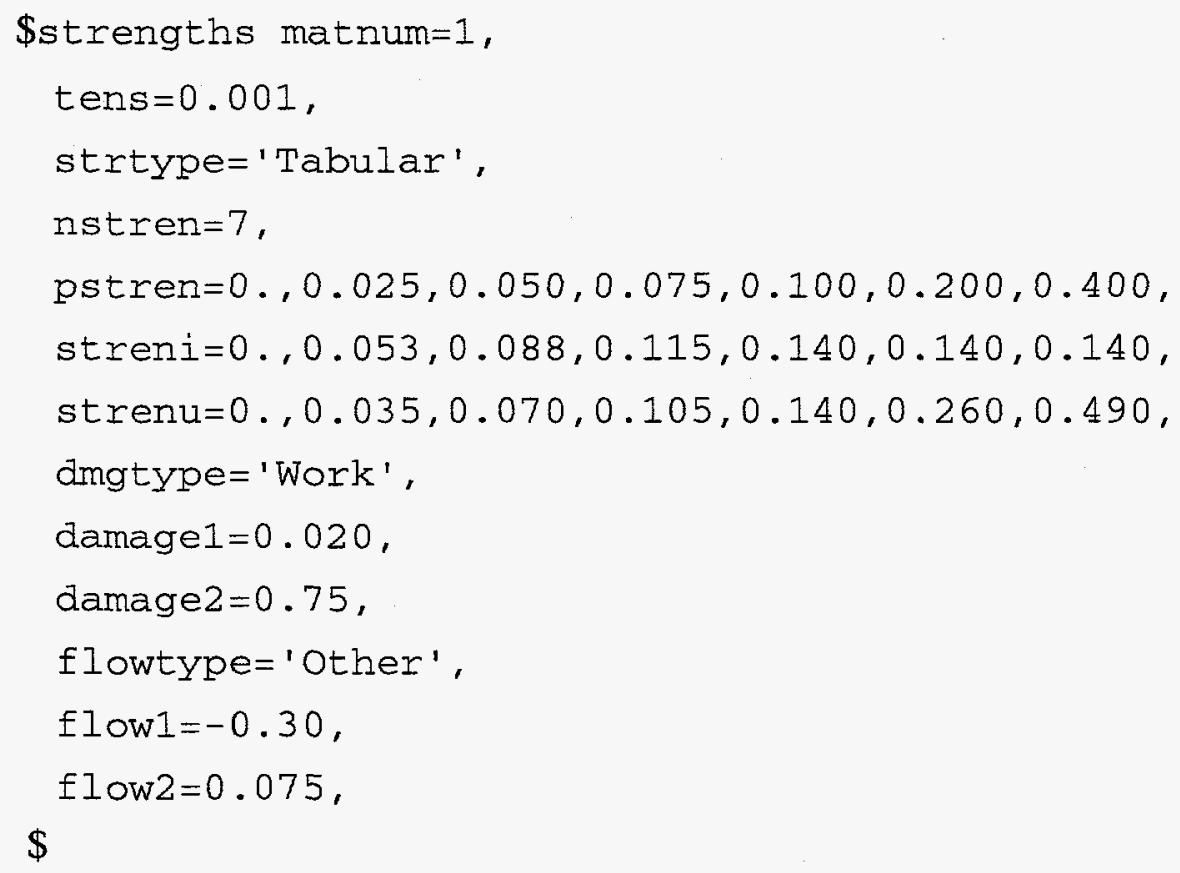

Other elastic constants for this material are specified in the following namelist:

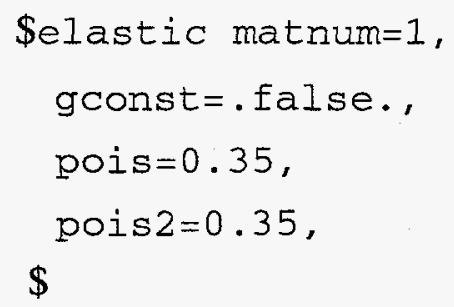




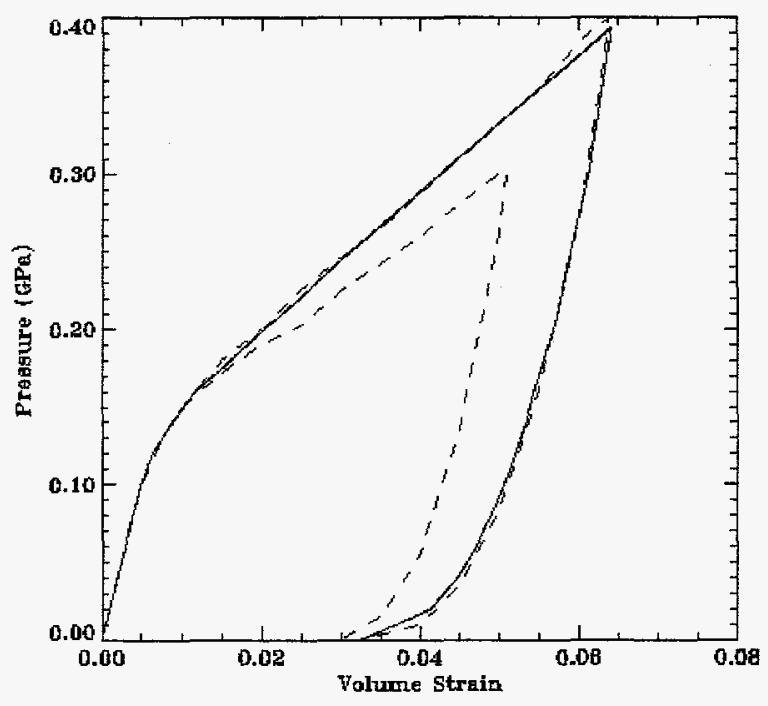

Figure 5. Pressure-volume data from drained, hydrostatic, quasistatic experiments (dashed lines) and model fit to data from one of the experiments (solid line). The differences between the experiments represent normal sample-to-sample variability of the rock.

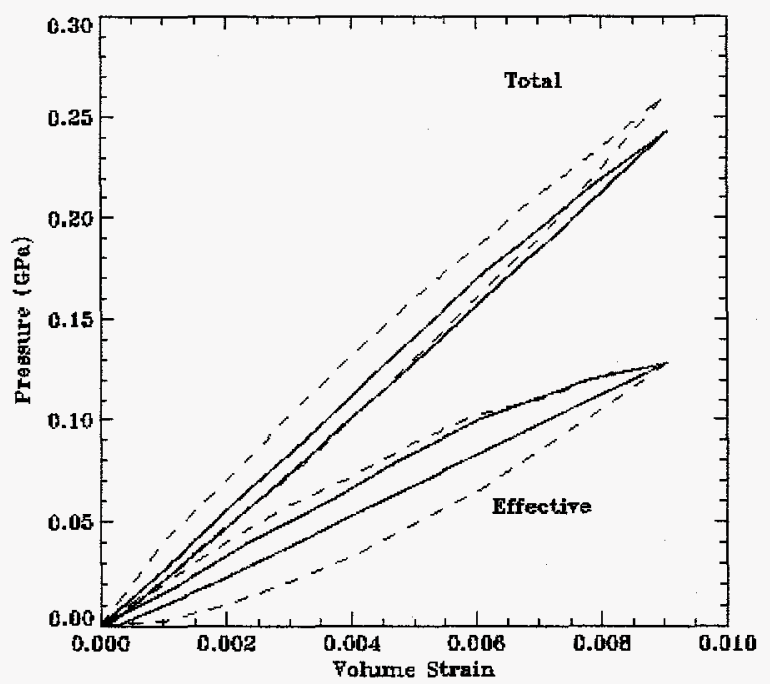

Figure 6. Pressure-volume prediction for fully saturated experiment (solid line) together with data from a corresponding laboratory experiment (dashed line).

\section{Example 2 - 1 kt explosion in fully and partially saturated limestone}

This example shows a 1-D spherically symmetric calculation of a $1 \mathrm{kt}$ explosion in a saturated limestone. The limestone model is the same as that used in Example 1. The partially saturated variation is specified simply by changing the value of the saturation parameter from 1.0 to 0.75 to specify $75 \%$ saturation. The initial stress conditions correspond to an overburden of $250 \mathrm{~m}$. For the fully saturated case the water table is also 
$250 \mathrm{~m}$ above the work point of the explosion. Thus the initial total pressure in the material is $6.2 \mathrm{MPa}$ and the initial pore pressure is $2.45 \mathrm{MPa}$ for the fully saturated example. For the partially saturated example the initial total pressure is $6.13 \mathrm{MPa}$, reflecting the slightly lower density of the partially saturated material. In the partially saturated example the pore pressure is initially zero while the initial air-filled void fraction is $2.75 \%$.

The explosion is modeled with a "pill" energy source; the explosion energy is deposited as internal energy in a $1 \mathrm{~m}$ radius pill of material at the center of the mesh. The simulation was run to $0.2 \mathrm{~s}$, by which time the ground shock had propagated to about 700 $800 \mathrm{~m}$ range and the explosion cavity had nearly reached its final dimensions. Figure 7 shows the peak principal overstress as a function of range for the two cases. At ranges greater than $20 \mathrm{~m}$, the additional dissipation due to the air-filled void has had most of its effect, and the peak stresses are roughly a factor of two smaller for the partially saturated case as for the fully saturated. The peak particle velocity, plotted in Figure 8 shows a similar result for the attenuation of the ground shock.

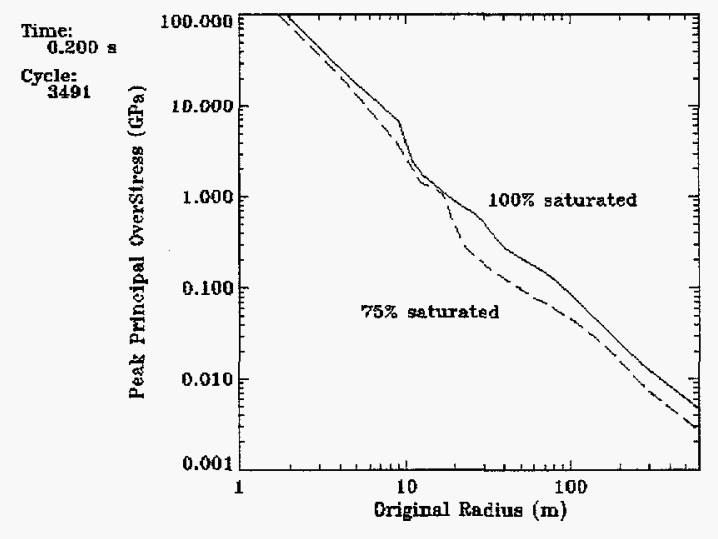

Figure 7. Peak overstress as a function of range for both calculations.

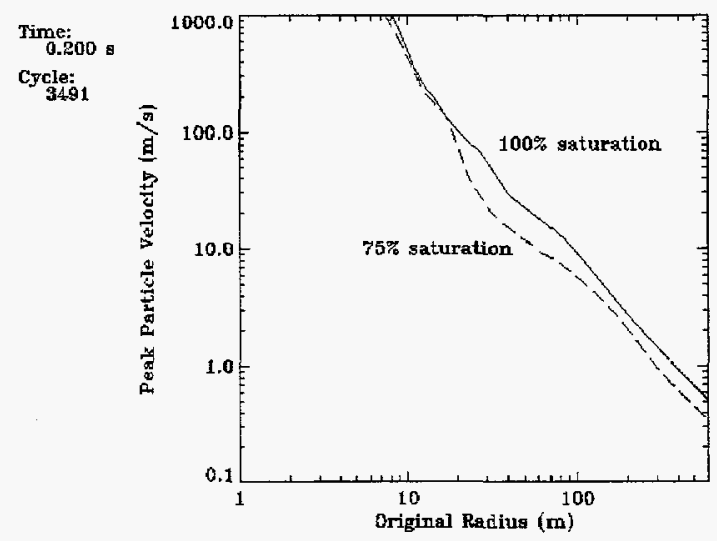

Figure 8. Peak particle velocity as a function of range for both calculations.

Figure 9 shows a snapshot of the pressure at $0.01 \mathrm{~s}$ for the fully saturated case. Note that at ranges around $25 \mathrm{~m}$, where the material is expanding from its maximum compression, the pore pressure equals the total pressure leading to a zero effective stress condition. A similar plot for the partially saturated case shows quite different behavior (Figure 10). The ground shock at this time has attenuated sufficiently so that the stresses are not capable of crushing out all the void space in this moderately strong rock. Consequently, no buildup in pore pressure is observed behind the shock front. Significant pore pressures are only observed closer to the working point, where the ground shock was substantially stronger and able to crush out all the void space and begin compressing the pore water. The change in behavior from fully to partially saturated conditions also leads to 
the formation of a larger residual stress field near the explosion cavity, which has a radius of slightly less than $10 \mathrm{~m}$ in this figure.

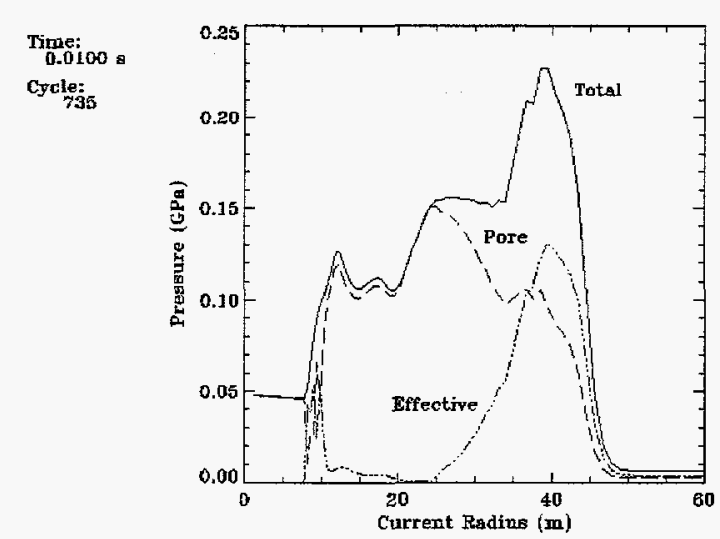

Figure 9.
Figure 9 is a snapshot of pressures at 0.01 $s$ for the $100 \%$ initial saturation case.

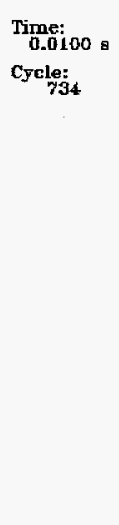

Figure 10.

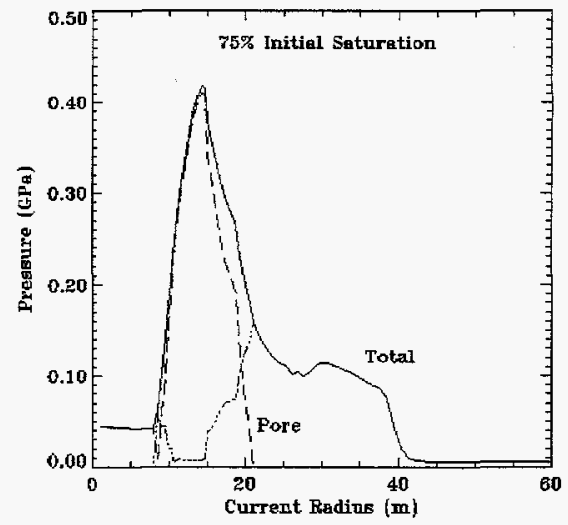

Figure 10 is a snapshot of pressures at $0.01 \mathrm{~s}$ for the $75 \%$ initial saturation case.

Figure 11 shows the value of the yield strength under the material conditions current at $0.01 \mathrm{~s}$ for the two cases. Regardless of saturation, the yield strength is a strong function of the effective pressure, so both cases show a substantial strength increase when the ground shock arrives and the effective pressure begins to increase.

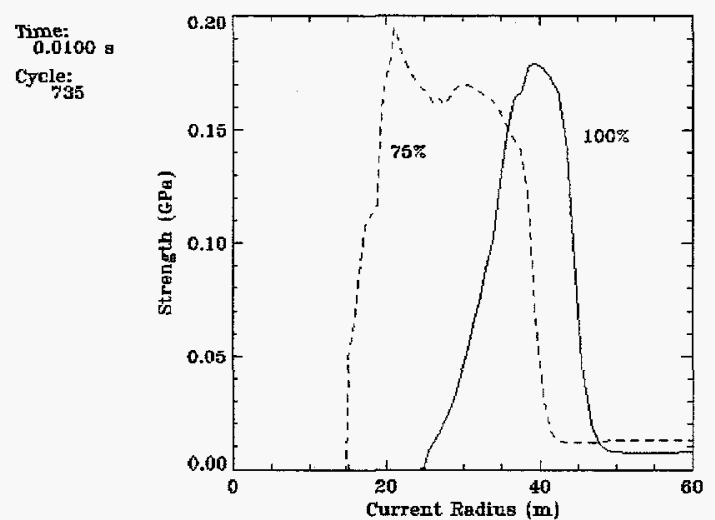

Figure 11. Snapshot of yield stress at $0.01 \mathrm{~s}$ for both saturation cases.

Just a short distance behind the shock front for the fully saturated case, the strength begins to decline, ultimately falling to zero. This reflects the tendency of a saturated or nearly saturated material to go to a liquefaction state after being sufficiently compressed to damage the pore structure. By contrast, the partially saturated case maintains a significant strength 
for all ranges from about $15 \mathrm{~m}$ to the shock front. It is only at ranges less than about $15 \mathrm{~m}$ that peak pressures damage the pore structure sufficiently allowing a liquefaction state to be reached.

\section{ACKNOWLEDGEMENTS}

This work was performed under the auspices of the U.S. Department of Energy.

\section{REFERENCES}

Biot, M.A., "General Theory of Three Dimensional Consolidation," J. Appl. Phys., $\underline{12}$, pp. 155-165, 1941.

Boettger, J.C. and S.B. Trickey, "Multiparameter Iterative Convergence Accelerator for Crystalline LCGTO Calculations," Comp. Phys. Comm., 32, pp. 361-365, 1984.

Brace, W.F., "Pore Pressure in Geophysics," in Flow and Fracture of Rocks, H.C. Heard, I.Y. Borg, N.L. Carter, C.B. Raleigh eds., Am. Geophy. Union, Washington, D.C., pp. 265-273, 1972.

Carroll, M.M. and A.C. Holt, "Static and Dynamic Pore-Collapse Relations for Ductile Porous Materials," J. Appl. Phys., 43, pp. 1626-1636, 1972.

Carroll, M.M., "Mechanical Response of Fluid-saturated Porous Materials," in Proc. 15th Int. Cong. on Theoretical and Applied Mechanics, F.P.J. Rimrott and R. Tabarrok eds., North Holland, NY, pp. 251-262, 1980.

Frederickson, A.A., H.D. Zimmerman, and J.A. Carney, "Effective Stress constitutive Models for Computational Applications," Defense Nuclear Agency Rept. DNA-TR-89-18, 1989.

Garg, S.K., "Wave Propagation Effects in a Fluid-Saturated Porous Solid," J. Geophys. Res., 76, pp. 7947-7962, 1971.

Garg, S.K., A.H. Nayfeh, and A.J. Good, "Compressional waves in fluid-saturated elastic porous media," J. Appl. Phys., 45, pp. 1968-1974, 1974. 
Garg, S.K., D.H. Brownell, J.W. Pritchett, and R.G. Herrmann, J. Appl. Phys., 46, pp. 702-713, 1975.

Handin, J., R.V. Hager, M. Friedman, and J.N. Feather, Bull. Am. Asso. Petrol. Geol., 47, pp. 717-755, 1963.

Herrmann, W., "Equation of State of Crushable Distended Materials," Sandia National Laboratories report SC-RR-66-2678, March 1968.

Jaeger, J.C. and N.G.W. Cook, Fundamentals of Rock Mechanics, 2nd edition, Halsted Press, New York, 1976.

Katsube, N., "The Constitutive Theory for Fluid-Filled Porous Materials," J. Appl. Mech., 52, pp. 185-189, 1985.

Katsube, N. and M.M. Carroll, "The Modified Mixture Theory for Fluid-Filled Porous Materials: Theory," J. Appl. Mech., 54, pp. 35-40, 1987.

Katsube, N. and M.M. Carroll, "The Modified Mixture Theory for Fluid-Filled Porous Materials: Applications," J. Appl. Mech., 54, pp. 41-46, 1987.

Kim, K.J., S.E. Blouin, D.E. Chitty, and D.H. Merkle, "Experimental and Theoretical Response of Multiphase Porous Media to Dynamic Loads," Applied Research Associates, South Royalton, VT, Sept. 1988.

Nur, A. and J.D. Byerlee, "An Exact Effective Stress Law for Elastic Deformation of Rock and Fluids," J. Geophys. Res., 76, pp. 6414-6419, 1971.

Sandler, I.S. and L. Whitman, "A Two-Phase Model of Fully Saturated Porous Geological Material," Defense Nuclear Agency Rept. DNA-TR-90-16, 1990.

Simons, D.A., "Comparison of Several Two-Phase Models for Saturated Porous Media," R\&D Associates Rept. RDA-TR-0264238903-001, 1989.

Swift, R.P. and D.E. Burton, "Numerical Modeling of the Mechanics of Nonlinear Porous Media Under Dynamic Loading," Lawrence Livermore National Lab. Rept. UCRL-53568, 1984. 
Terzaghi, K., Theoretical Soil Mechanics, Wiley, New York, NY., 1943.

Wilkins, M.L., "Calculation of Elastic-Plastic Flow," in Methods in Computational Physics, B. Alder. S. Fermbach, and M. Rotenberg, Eds., Academic Press, New York, pp. 211-264, 1964. 


\section{APPENDIX A - SMC123 IMPLEMENTATION}

Input parameters for effective stress model in SMC123.

The input parameters are specified in three namelists. The "effstress" namelist contains parameters for the volumetric portion of the model. The "elastic" namelist contains the shear modulus or Poisson's ratios. The "strengths" namelists contains the tensile and shear failure parameters, including strain hardening/softening parameters, strain rate dependence, and flow rule.

effstress

c Specify parameters for effective stress model

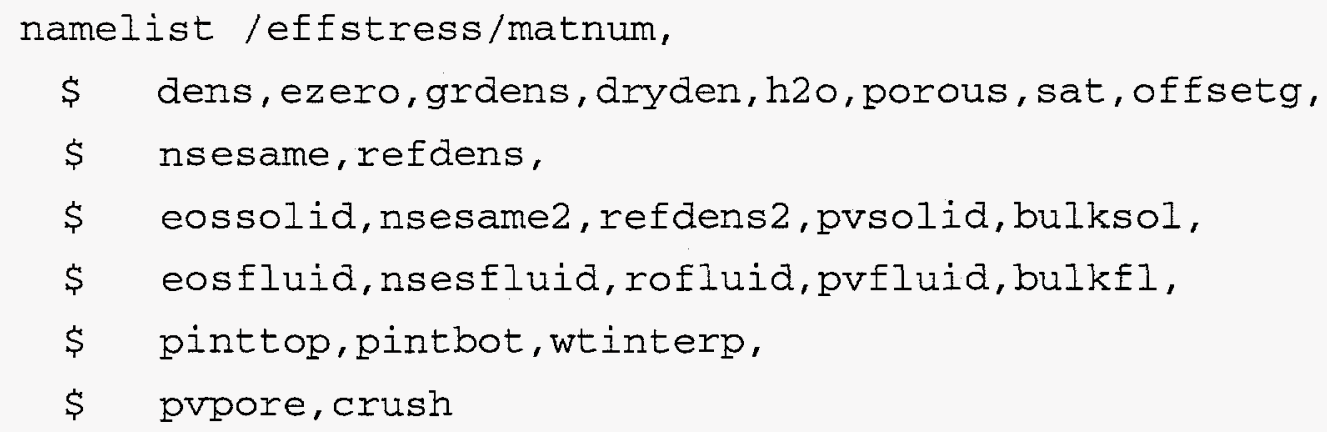

matnum - Material number.

Density, porosity and saturation.

Specify one of the following three sets:

Set X

dens - Initial density (udensity).

grdens - Grain density (udensity).

h2o - Water weight fraction.

Set $\mathrm{Y}$

dryden - Dry bulk density (udensity).

porous - Porosity.

sat - Relative saturation.

Set Z

grdens - Grain density (udensity).

porous - Porosity.

sat - Relative saturation. 
ezero - Initial specific energy (uedense).

Solids equation of state eossolid - String specifying type of solid eos.

Valid types are 'linear' or 'Linear', 'tabular' or 'Tabular' or 'sesame' or 'Sesame'

refdens2 - Reference density for solids Sesame table (udensity). bulksol - Solid bulk modulus for 'linear' solid EOS (ustress) pvsolid - File containing tabular P-V/V0 curves for solids. nsesame2 - Sesame table number of 'sesame' solid EOS

Fluids equation of state eosfluid - String specifying type of fluid eos.

Valid types are 'linear' or 'Linear', 'tabular' or 'Tabular' or 'sesame' or 'Sesame' rofluid - Reference density for fluid ( $\mathrm{def}=1.0 \mathrm{gm} / \mathrm{cc}$ )(udensity) bulkfl - Fluid bulk modulus for 'linear' solid EOS (ustress) pvfluid - File containing tabular P-V/V0 curves for fluid. nsesfluid - Sesame table number of 'sesame' fluid EOS

Porosity crushing relationship pvpore - File containing effective pressure-pore crushing curves. wtinterp - Exponent on weighting factor for interpolating an unload/reload curve $(\mathrm{def}=1.0)$

SESAME table nsesame - Sesame table number for use for total material that has reached a peak pressure in excess of pintbot. refdens - Reference density for Sesame table (udensity). pinttop - Upper pressure for merging $\mathrm{p}-\mathrm{V}$ behavior into Sesame table (ustress).

pintbot - Lower pressure for merging $\mathrm{p}-\mathrm{V}$ behavior into Sesame table (ustress).

elastic

Specify elastic parameters for most models and linear elastic material 
namelist /elastic/matnum, dens,

$\$$ pois, pois2, pwave, youngs, bulk, shearmod, gconst

matnum - Material number.

dens - Initial density (udensity).

Elastic moduli.

pois - Poisson's ratio for loading.

pois2 - Poisson's ratio for unloading/reloading.

pwave - P-wave velocity (uveloc).

youngs - Young's modulus (ustress).

bulk - Bulk modulus (ustress).

shearmod - Shear modulus (ustress).

gconst - Flag for holding shear modulus constant during unload/reload.

strengths

c Specify shear and tensile strength parameters for all models.

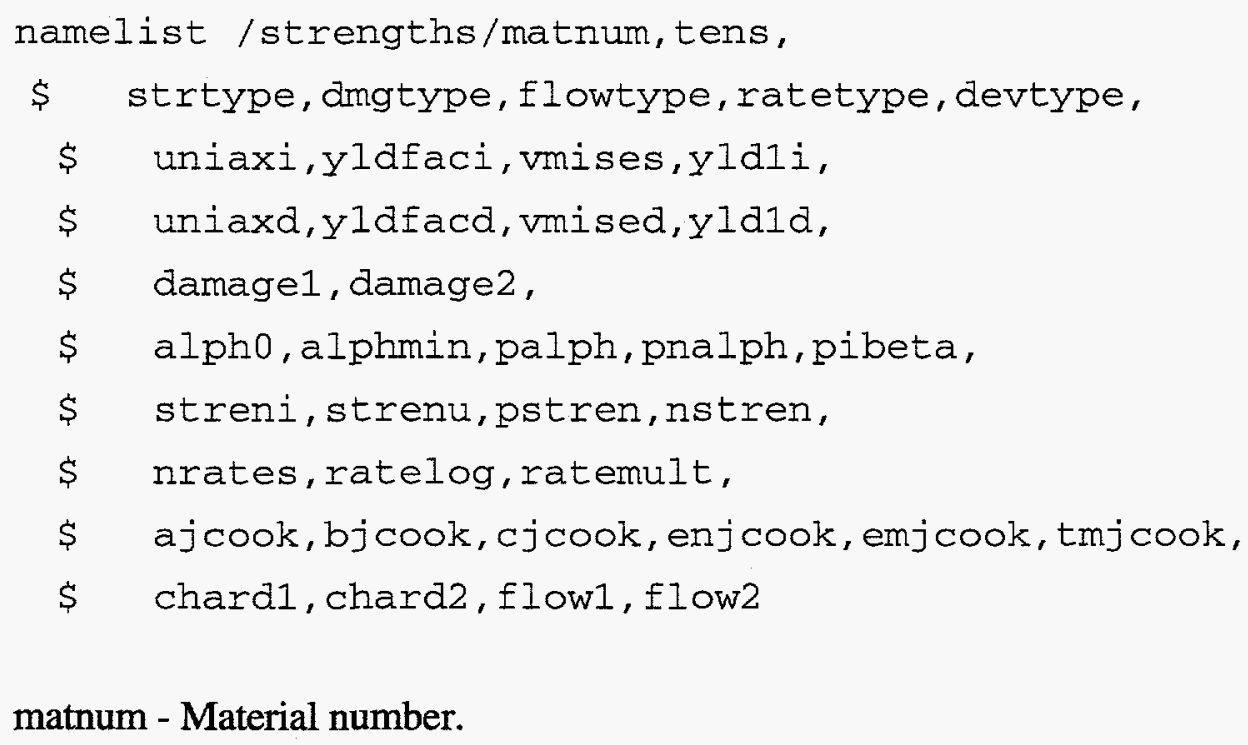

matnum - Material number.

Tensile strength.

tens - Tensile strength (ustress).

Type of strength model 
strtype - Type of strength model. Valid types are:

'Johnson-Cook' or 'johnson-cook',

'Tabular' or 'tabular',

'Exponential' or 'exponential', and

'None' or 'none'

Johnson-Cook strength model (strtype='Johnson-Cook' or 'johnson-cook')

(strength $=($ ajcook + bjcook $*$ (plastic strain $) * *$ enjcook $) *($

$\left(1 .+\right.$ cjcook $^{*} \ln ($ dev. strain rate) $) *(1 .-($ homologous temp)**emjcook)

ajcook - Initial strength at room conditions and strain rate of $1 / \mathrm{s}$ (ustress).

bjcook - Hardening parameter (ustress).

enjcook - Exponent for strain hardening dependence.

cjcook - Rate parameter

emjcook - Exponent for temperature dependence.

tmjcook - Melting temperature (utemper).

Exponential shear strength function.

Strength=vmises-yld $1 * \exp (-$ pmean/yldfac $)$

uniaxi - Uniaxial (unconfined) compressive strength (ustress).

vmises - Asympototic max. stress difference (ustress).

yldfaci - See above equation (ustress).

yld1i - See above equation (ustress)

uniaxd - Ultimate uniaxial compressive strength (ustress)

vmised - Ultimate asympototic max stress difference (ustress).

yldfacd - Ultimate value of yldfac above (ustress).

yld1d - Ultimate value of yld1 above (ustress).

Tabular shear strength model.

streni - Array of initial max. stress differences (ustress).

strenu - Array of ultimate max. stress differences (ustress).

pstren - Array of mean stress corresponding to above (ustress).

nstren - Number of entries in strength arrays.

Flow rule.

flowtype - Type of flow rule. Types are:

'Prandtl' or 'prandtl' for Prandtl-Reuss (default),

'Associated' or 'associated', and 
'Other' or 'other' for a special non-associated rule.

flow1 - First parameter in special flow rule.

flow2 - Second parameter in spcial flow rule.

Work hardening and softening.

Hardening based on equivalent plastic strain or plastic work.

dmgtype - Type of damage function. Types are

'Linear' or 'linear': linear function of plastic strain,

'Exponential' or 'exponential': exponential function of plastic strain,

'Work' or 'work': exponential function of plastic work.

'None' or 'none': no damage assumed (default)

damage 1 - First parameter in damage function.

damage 2 - Second parameter, if needed, in damage function.

Strain rate dependence of strength (Total, not plastic strain)

ratetype - Type of strain rate dependence. Types are

'Tabular' or 'tabular' - Use table below

'None' or 'none' - No rate dependence (default)

nrates - Number of entries in table

ratelog - Array of $\log 10$ of deviator strain rate

ratemult - Array of multipliers of strength value.

Noncircular shape of failure surface in deviator space

devtype - Turns on non circular failure envelope in pi plane

'Devshape' or 'devshape' or 'devshape-model'

'None' or 'none' - Circular shape (default)

alpha=alph $0 *(1-\text { pmean/palph })^{* *}$ pnalph for pmean $<=$ palph

alph0 - Shape factor in range 0-1 (1: triangular, 0 : circular)

alphmin - Value of alpha for pmean >palph

palph - Pressure factor in above formula (ustress)

pnalph - Exponent on pressure term 


\section{APPENDIX B - CTH IMPLEMENTATION}

\section{Routines for LANL effective stress model in CTH.}

This implementation was done using the 1995 version CTH version. Because six internal state variables are used by the model, the user should not use this model for more than three material numbers. This version of $\mathrm{CTH}$ has limited additional array space for internal state variables. Because the code does not store tables supplied by the user with the DEFTABLE command in the dump, the tables required for the volumetric part of the model must appear in both the CTHGEN and CTH sections of the input file.

The following tables show which subroutines and modules are affected by this implementation.

$\begin{array}{ll}\text { Modules } & \text { Subroutines } \\ \text { elsg.for } & \text { ELSG } \\ \text { elygp.for } & \text { ELYGP } \\ \text { eos.for } & \text { EOS } \\ \text { eosgen.for } & \text { EOSGEN } \\ \text { eosmap.for } & \text { EOSMAP + all new subroutines } \\ \text { gcntrl.for } & \text { GCNTRL } \\ \text { uinep.for } & \text { UINEP } \\ \text { uinisv.for } & \text { UINISV } \\ \text { fracp.for } & \text { FRACP } \\ \text { fracs.for } & \text { FRACS } \\ \text { prelax.for } & \text { PRELAX } \\ \text { ptrelx.for } & \text { PTRELX } \\ \text { incavf.all } & \text { Includes for avf developer library. }\end{array}$

Replacement routines for existing CTH routines

ELSG - Computes new deviator stresses and applies yield criteria.

Add call to ELVPEF.

ELYGP - Gets the shear modulus for each material.

Add call to GETGEE

EOS - Calls all the EOS routines at startup to initialize models.

Add call to EOSEFF, used only during setup

EOSGEN - Reads in EOS data from input file.

Add call to EFFSTI 
EOSMAP - Call all the appropriate EOS routines for the regular EOS computations during the course of a calculation.

Add call to EOSEFV, for regular EOS calls during the calculation

GCNTRL - Moved where user defined tables are read to an earlier point in the routine.

UINEP - Read in elastic-plastic data.

Add most effective stress model input parameters

UINISV - Allocate space for internal state variables for various models. Add effective stress model internal state variables

FRACP - No coding change, just needs opportunity to recompile with new INCLUDE EOSVECT block.

FRACS - No coding change, just needs opportunity to recompile with new INCLUDE EOSVECT block.

PRELAX - No coding change, just needs opportunity to recompile with new INCLUDE EOSVECT block.

PTRELX - No coding change, just needs opportunity to recompile with new INCLUDE EOSVECT block.

incavl.all - Added EOSEFV call to INCLUDE EOSVECT block.

New Routines

EFFSTI - Read in required solids and fluid SESAME tables for effective stress model. This is a specially modified version of SESESI.

ELVPEF - Compute current strength and also solve for flow rule effects.

EOSEFV - Driver for regular EOS calls for effective stress model

EOSEFF - Driver for EOS initialization for effective stress model.

FLUIDEOS - Driver for EOS of fluid. Called by SOLVE

GETGEE - Compute current shear modulus for effective stress model

ISRCHK - Utility for searching in tables. Called by FLUIDEOS, POREEOS and SOLIDEOS.

POREEOS - Interpolates effective pressure-porosity behavior from tables.

Called by SOLVE and GETGEE.

SOLIDEOS - Driver for EOS of solid. Called by SOLVE and GETGEE.

SOLVE - Solve effective pressure equations for volumetric behavior.

Called by EOSEFV and EOSEFF.

Input parameters for the LANL effective stress model in CTH 
EOS parameters

EFFSTR - Keyword for this material model

EOS_FLUID - Sesame table number for fluid eos

FEOS_FLUID - File containing Sesame table for fluid

EOS_SOLID - Sesame table number for solids eos

FEOS_SOLID - File containing Sesame table for solid

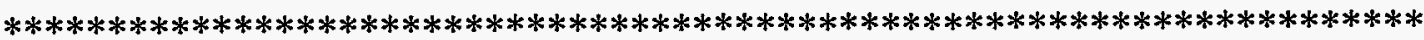

EPDATA parameters

Basic parameters

EFFSTR - Keyword for this material model

To specify the density and fluid saturation conditions choose either the

set: EFF_DENS, EFF_POROUS, EFF_FLUIDW

or the set: EFF_DRYDEN, EFF_POROUS, EFF_SAT

EFF_DENS - Initial density of material

EFF_FLUIDW - Initial fluid weight fraction

EFF_DRYDEN - Initial dry density of the material

EFF_SAT - Initial fluid saturation of the material

EFF_POROUS - Initial porosity of material

\section{Effective pressure-normalized porosity tables}

User must supply tables for both an initial crush curve and an unload curve from the highest point on the initial crush curve using DEFTABLE numbers 101-109 for the initial crush curve and numbers 111-119 for the master unload curve. EFF_WTINTERP - Interpolation exponent for constructing unload/reload curves from initial crush and master unload porosity curves (default $=1.0$ )

\section{Fluid model}

EFF_IFLUID - Type of fluid eos:

0 : Constant bulk modulus 
1: User supplied table

2: Sesame table - define the table number in the EOS input

EFF_ROFLUID - Initial density of pore fluid (default $=1.0 \mathrm{gm} / \mathrm{cm}^{3}$ )

EFF_BULKFL - Bulk modulus for fluid for EFF_IFLUID=1

\section{Solid model}

EFF_ISOLID - Type of solid eos:

0 : Constant bulk modulus

1: User supplied table

2: Sesame table - define the table number in the EOS input

EFF_BULKSOL - Bulk modulus for solid for EFF_ISOLID=1

\section{Other elastic parameters}

Choose either the Poisson's ratios or the shear modulus

EFF_POIS1 - Poisson's ratio during initial crushup

EFF_POIS2 - Poisson's ratio during unload/reload

EFF_SHEARMOD - Drained shear modulus

\section{Yield surface}

This model requires both an initial and an ultimate shear yield surface

EFF_ISTREN - Type of yield envelope:

0 : No strength

1: Exponential function, $\mathrm{Y}=\mathrm{Y} 0-\mathrm{Y} 1 * \exp (-\mathrm{Peff} / \mathrm{Y} 2)$

2: User supplied tables, supply both an initial and ultimate table of yield stress as a function of effective pressure.

For initial yield surface, use DEFTABLE numbers 141-149.

For ultimate yield surface, use DEFTABLE numbers 151-159.

The last digit corresponds to the material number.

EFF_VMISES - Initial value of Y0 in exponential function

EFF_VMISED - Ultimate value of Y0 in exponential function

EFF_YLD1I - Initial value of Y1 in exponential function

EFF_YLD1D - Ultimate value of Y1 in exponential function

EFF_YLDFACI - Initial value of Y2 in exponential function

EFF_YLDFACD - Ultimate value of Y2 in exponential function

Evolution of yield surface from initial to ultimate. Also evolution 
of tensile strength from initial value to zero.

EFF_IDMG - Type of "damage" to yield curves:

0 : No damage - use initial yield surface throughout

1: Linear function of equivalent plastic strain (EQPSO)

factor=min(1,max (0,(EQPSO-EFF_DMG1)/(EFF_DMG2-EFF_DMG1))

2: Exponential function of equivalent plastic strain factor=1.0-exp(-EQPSO/EFF_DMG1)

EFF_DMG1 - Coefficient for linear and exponential damage functions

EFF_DMG2 - Coefficient for linear damage function

Strain rate dependence of shear and tensile yield surfaces.

EFF_IRATE - Type of strain rate dependence of yield surface

0 : No strain rate dependence

1: User supplied table, supply multiplying factor as function

of $\log 10$ of deviatoric strain rate

Use DEFTABLE numbers 161-169.

The last digit corresponds to the material number.

Flow rule

EFF_IFLOW - Type of flow rule:

0: Prandtl-Reuss flow rule

1: Associated flow rule

2: A non-associated, non-Prandtl-Reuss, flow rule slope2=EFF_FLOW1+EFF_FLOW $2 *$ (damage factor)

EFF_FLOW1 - Coefficient for non-associated flow rule

EFF_FLOW2 - Coefficient for non-associated flow rule

Shape of yield surface in pi plane

EFF_ALPHA - Shape coefficient (0.0: circular, 1.0: triangular)

Table numbers for user supplied tables.

Supply these tables with the DEFTABLE command. Tables for effective-pressure-porosity and the fluid and solid PV curves must be defined in both the CTHGEN and the CTH sections of your input file. The other tables must be defined in the CTH section.

(the final digit corresponds to the material number)

101-109 : Initial peff-phi/phi0 crush curve 
111-119: Unload peff-phi/phi0 crush curve

121-129 : Fluid P-V/V0 curve

131-139 : Solid P-V/V0 curve

141-149 : Initial strength

151-159 : Ultimate strength

161-169: Strain rate dependence

Internal State Variables

Six internal state variables are used by the model. The equivalent plastic strain is used for the strain hardening/softening rule in both the tensile and shear strength models. The pore pressure is computed in the volumetric part of the model and used in both the volumetric and the deviatoric parts of the model. The minimum normalized porosity is used to capture one element in the history of the material and used in constructing the unload/reload volumetric behavior. The porosity is computed in the volumetric part of the model and is carried from cycle to cycle to give a good starting point for the solver. The shear-induced porosity change records the feedback effect of shear-enhance void collapse and dilatancy on the volumetric part of the model. The fluid internal energy is the specific internal energy of the fluid and captures the effect that the fluid is often compressed more than the solid phase.

Equivalent Plastic Strain - This is the same measure of plastic strain as is used in the Johnson-Cook strength model.

For CTHPLT specify: "Qn" where $\mathrm{n}$ is the material number.

For HISPLT specify: "PL-STRAIN-MATn", where $n$ is the material number.

Pore Pressure - The current value of the pore fluid pressure.

For CTHPLT specify: "XPPn" where $\mathrm{n}$ is the material number.

For HISPLT specify: "PORE-PR-MATn", where $\mathrm{n}$ is the material number.

Minimum normalized porosity - The minimum porosity ever experienced by the material.

This value is normalized by the initial porosity of the material.

For CTHPLT specify: "XPHMn" where $\mathrm{n}$ is the material number.

For HISPLT specify: "MIN-PHI-MATn", where $\mathrm{n}$ is the material number.

Porosity - The current value of the porosity.

For CTHPLT specify: "XPHIn" where $\mathrm{n}$ is the material number. 
For HISPLT specify: "POROSITY-MATn" where $\mathrm{n}$ is the material number.

Shear-induced porosity change - The net increase or decrease of the total porosity due to shear-enhanced void collapse and dilatancy.

For CTHPLT specify: "XSCPn" where $\mathrm{n}$ is the material number.

For HISPLT specify: "SHR-PHI-MATn" where $\mathrm{n}$ is the material number.

Fluid Specific energy - The specific internal energy of the pore fluid.

For CTHPLT specify: "XFEn" where $\mathrm{n}$ is the material number.

For HISPLT specify: "FLUID-E-MATn" where $\mathrm{n}$ is the material number. 
